doi.org/10.22498/pages.29.1.21

\title{
Fabulous interglacials: A timeline of the PIGS and QUIGS working groups
}

\author{
Chronis Tzedakis', L. Menviel², E. Capron³, B.L. Otto-Bliesner', J.F. McManus ${ }^{5}$, \\ D. Raynaud ${ }^{3}$ and E. Wolff 6
}

Part of the scientific rationale for pursuing studies of past interglacials is that they provide a baseline against which to assess the climatic evolution of the current interglacial and the impact of anthropogenic interference. Here, we trace the history of the PAGES working groups on interglacials (PIGS and QUIGS).

\section{Prelude}

When we look at the past, our attention is often captured by the allure of the recent (the last couple of millennia) or the shock of the extreme (a planet plunging into an ice age). However, although the past provides no exact analog for the next century and beyond, it is interglacials that provide examples that are most relevant for assessing the current anthropogenic warming, including its influence on the cryosphere and the feedbacks associated with biogeochemical cycles. For that reason, the study of the full range of past interglacials, their climate variability and impacts, their initiation and their ending, are a cornerstone of PAGES' research.

\section{PIGS}

On the evening of 30 January 2007, Jerry McManus, Dominique Raynaud and Chronis Tzedakis were talking quietly in a corner

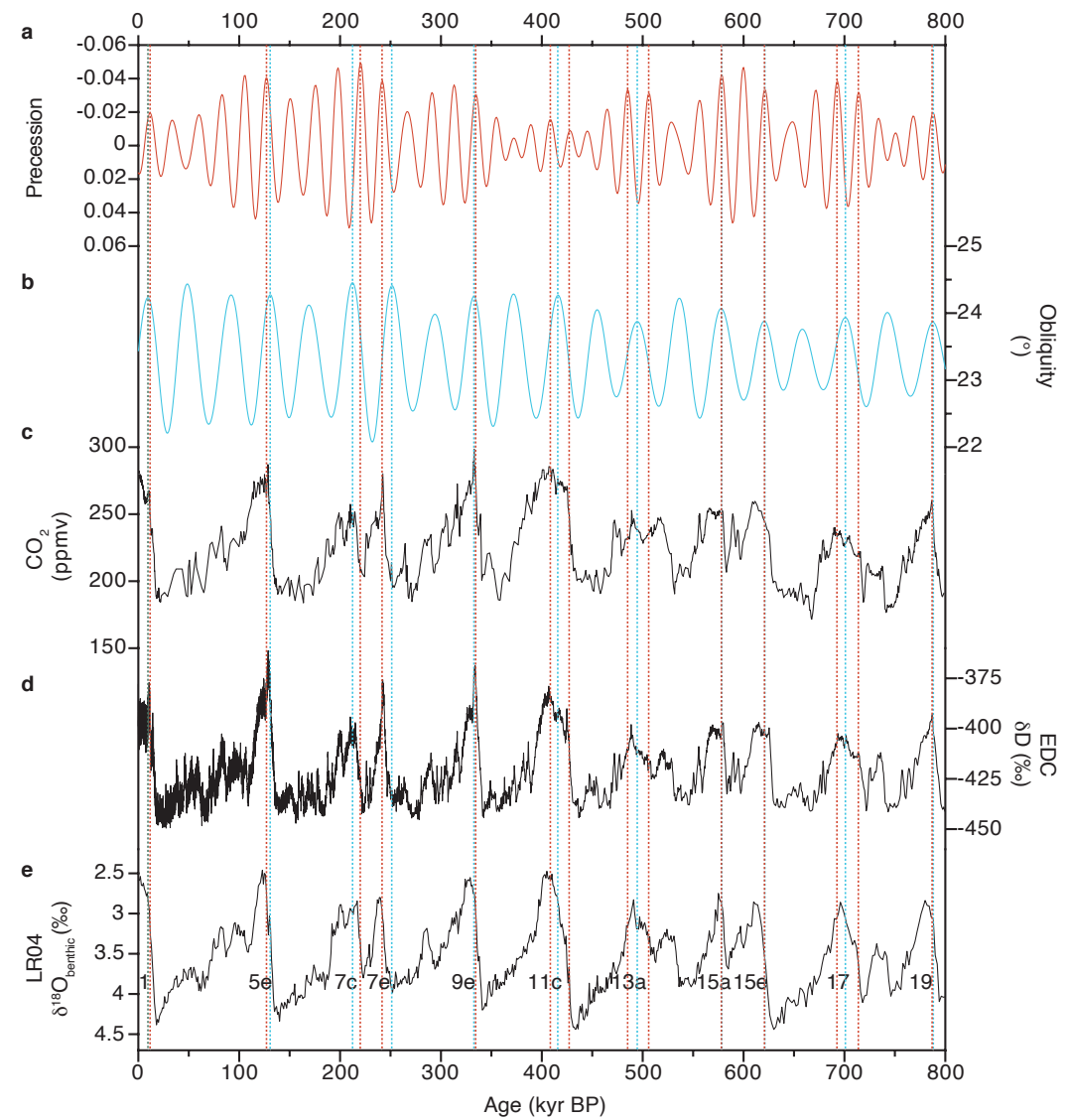

Figure 1: Interglacials of the last 800 kyr. (A) Precession parameter, plotted on an inverse vertical axis (Berger 1978). (B) Obliquity (Berger 1978). (C) Atmospheric $\mathrm{CO}_{2}$ concentration from Antarctic ice cores (Lüthi et al. 2008). (D) $\delta D$ of ice in the EPICA EDC ice core, Antarctica (Jouzel et al. 2007). (E) $\delta^{18} \mathrm{O}_{\text {benthic }}$ record from the LR04 stack (Lisiecki and Raymo 2005). Marine isotope stages and substages of interglacial status are shown. Vertical dotted lines indicate the timing of precession minima (red) and obliquity maxima (blue). Note the three longer interglacials (MIS 11c, 13a, 17) where precession and obliquity are nearly opposite in phase (Tzedakis et al. 2012). Modified from Tzedakis et al. (2009).

of the Captain Kidd bar at Woods Hole Village, on Cape Cod, MA, USA. It had been a good scientific meeting. After two days of talks on Marine Isotope Stage (MIS) 11, the workshop organized by McManus was drawing to a close. In fact, the entire working group on MIS 11, sponsored by the International Union for Quaternary Research (INQUA; inqua.org), was drawing to a close. "Where do we go from here?" the trio asked. "Perhaps we could learn more, if we looked at the whole ensemble of interglacials in a systematic way," said Raynaud. Not entirely clear-headed, they called it "PIGS" for Past Interglacials, and the name stuck pigs/intro).

They decided to approach PAGES for sponsorship. In its earlier days, PAGES had been focused primarily on the Holocene, but it was now under new management. Paleoceanographer Thorsten Kiefer had recently become the executive director and was possibly amenable to expanding the scope of PAGES to longer timescales. The plan for PIGS was to focus on the last 800 thousand years (kyr), as Antarctic ice-cores were furnishing information on atmospheric concentrations of greenhouse gases over that interval, thereby providing constraints on global boundary conditions. Kiefer was indeed receptive to such a prospect, and later in 2007 a proposal for a PAGES working group on Past Interglacials was accepted by the PAGES Scientific Steering Committee (SSC).

The first PIGS workshop (2-4 October 2008; pastglobalchanges.org/calendar/past/2008past/127-pages/1024) assessed the then state of knowledge and defined specific priority topics that would form the agenda of subsequent workshops. A PIGS-community progress article (Tzedakis et al. 2009) pointed to the large diversity among interglacials in terms of their intensity, structure, and duration (Fig. 1), but also noted that a general theory accounting for this remained elusive. In essence, an underlying ambition of all PAGES interglacial working groups has been to elucidate some general principles governing this diversity.

The second PIGS workshop (24-27 August 2009; pastglobalchanges.org/calendar/ past/2009-past/127-pages/1023) proved a resounding success and failure, in equal measure. Over the course of four days, it showed the full potential of small group meetings ( 30 participants), as an interglacial community of like-minded scientists began to form, freely exchanging ideas, showing each other unpublished data, and intensely debating general issues and finer points. A modus operandi emerged where community papers would be planned, but otherwise participants were free to pursue their own avenues of research independently, energized by the discussions at the meetings. This caused some consternation at PAGES headquarters, as some papers never formally acknowledged PIGS, although others did (e.g. Mokeddem et al. 2014), but overall the science moved forward. One of the ideas pursued during the meeting was the role of millennial-scale variability as an intrinsic feature of the past five glacial terminations, and plans were made for a PIGS-community paper on this topic. All the initial excitement, however, evaporated a few weeks later, after 
a brilliant paper on Ice Age Terminations was published by a different group (Cheng et al. 2009), making essentially the same point. The PIGS paper was abandoned.

The third PIGS workshop (20-22 October 2010; pastglobalchanges.org/calendar/ past/2010-past/127-pages/1022) focused on interglacial duration and glacial inception. Although estimates of interglacial durations are sensitive to the definition of interglacial conditions in different proxies and archives, it was thought that robust patterns could emerge from a systematic comparison of interglacials. One outcome was a paper (Tzedakis et al. 2012) arguing that the fundamental concept underlying the terminology of an interglacial is that of the sea-level highstand, a measure of integrated global climate effects that lead to the loss of continental ice; by extension, interglacial length was linked to the duration of the highstand. On this basis, it suggested that over the last $800 \mathrm{kyr}$, the phasing of precession and obliquity influenced the persistence of interglacial conditions over one or two insolation peaks, leading to shorter ( 13 kyr) and longer ( 28 kyr) interglacials (Fig. 1).

The fourth PIGS workshop (2-5 July 2012; pastglobalchanges.org/calendar/past/2012past/127-pages/1012) focused on how well we can explain the diversity of interglacials from the forcing and feedbacks and attempted to place interglacials within the wider context of ice-age cycles and the extent to which these are deterministic. A community paper to develop the major themes considered over the course of PIGS was planned, and a followup writing workshop brought together the lead authors for each section of the paper at Louvain la Neuve, Belgium, in March 2013. Eric Wolff took up the gargantuan task of editing and putting the different sections together. The landmark paper "Interglacials of the last 800,000 years" appeared in 2016 (Past Interglacials Working Group of PAGES
2016), condensing in 58 pages the then state of knowledge. It proposed that an objective definition of an interglacial is the absence of substantial Northern Hemisphere ice outside Greenland. A corollary of this is the occurrence of more than one interglacial within MIS 7 and MIS 15 (Fig. 1). Thus, interglacials of the past $800 \mathrm{kyr}$ do not occur every 100 $k y r$, and therefore attempts to predict the onset of interglacials need to account for this irregular return time. The review corroborated the crucial role that millennial-scale climate change (involving rapid changes in Atlantic Meridional Overturning Circulation strength) plays in each glacial termination. It highlighted MIS $5 \mathrm{e}$ as the interglacial that experienced the warmest conditions of the last 800 kyr across the globe. Taking a look into the future, the paper concluded that the next glacial inception is unlikely to occur in the next $50 \mathrm{kyr}$, given the combined effect of the current low eccentricity and high atmospheric greenhouse gas concentrations.

\section{QUIGS}

The PAGES-PMIP Working Group on Quaternary Interglacials (QUIGS; pastglobalchanges.org/quigs) arose from an initiative of Bette Otto-Bliesner, who envisaged a more formal connection between the successor to PIGS and the Paleoclimate Modelling Intercomparison Project (PMIP; https://pmip.Isce.ipsl.fr). More specifically, QUIGS would promote a closer collaboration between modelers and the data community to provide expertise on experimental design, data compilations and model-data comparisons, and to assess the relevance of interglacials to understanding future climate change.

With the guidance of PAGES SSC members Hubertus Fischer and Michal Kucera, an ambitious QUIGS working group structure, comprising two three-year QUIGS phases with a one-year gap, was envisaged: in Phase 1, QUIGS would formulate research

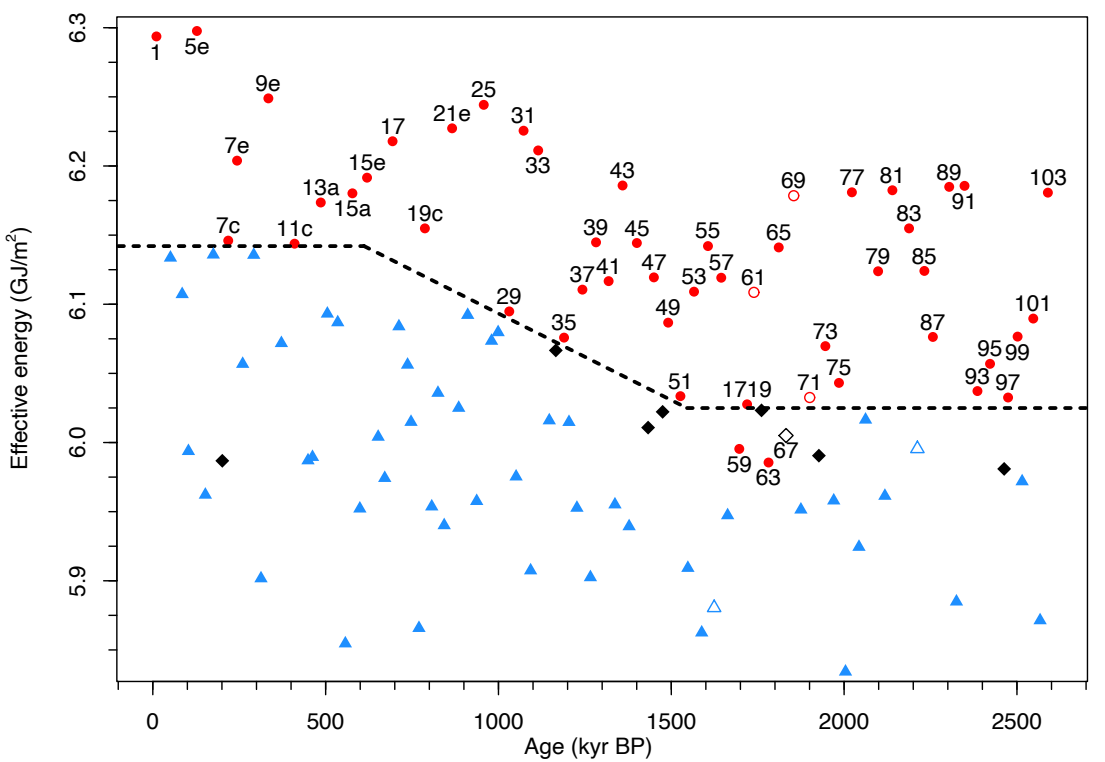

Figure 2: Effective energy for deglaciation (= peak caloric summer insolation + [(time elapsed since interglacia onset) $\times$ (discount rate)]) at each insolation peak during the past $2.6 \mathrm{Myr}$. Each insolation peak is plotted as the onset of an interglacial (red circles), a continued interglacial (black diamonds) or an interstadial (light blue triangles); open symbols correspond to uncertain assignments. The dotted line separates complete deglaciations from incomplete and missed deglaciations; the ramp indicates a gradual rise in the threshold required for a complete deglaciation. Numbers refer to Marine Isotope Stages. Modified from Tzedakis et al. (2017). questions, identify knowledge gaps, and plan how to fill these gaps. In close collaboration with PMIP, it would define model protocols and initiate the model runs and data collection needed. In Phase 2, the working group would return to the research questions identified in Phase 1, with better datasets and new model experiments, ultimately aiming to gain a quantitative understanding of interglacial controls. The four-year return period (between the beginning of Phase 1 and the beginning of Phase 2) would therefore provide the necessary time to complete the tasks.

QUIGS, led by Bette Otto-Bliesner, Emilie Capron, Anne de Vernal, Eric Wolff, and Chronis Tzedakis, was formally approved by the PAGES SSC in May 2015. A little later, Andrea Dutton, Anders Carlson, and Laurie Menviel joined the team.

The first QUIGS workshop on Warm Extremes (9-11 November 2015; pastglobalchanges.org/calendar/2015/127 pages/1520) assessed the current knowledge and research needs on the temporal and spatial patterns of climate forcing, responses, and feedbacks during MIS $5 \mathrm{e}$ and MIS 11. Paleorecords and climate model simulations highlighted the need for an improved understanding of the magnitude and drivers of the enhanced warmth during MIS $5 e$ and 11. This led to the definition of model protocols for CMIP6 and PMIP4 Last Interglacial simulations (Otto-Bliesner et al. 2017), and also of surface-climate data benchmarks for high-latitude regions (Capron et al. 2017).

The second QUIGS workshop (18-20 October 2016; pastglobalchanges.org/ calendar/2016/127-pages/1592) examined patterns of climate forcing, feedbacks, and responses characterizing glacial terminations. It assessed common features and differences between Terminations I and II ( $\mathrm{TI}$ and $\mathrm{TII}$ ), and highlighted the need for improved chronologies and for constraining the size and spatial distribution of ice sheets during the penultimate glacial maximum. This led to an article presenting a protocol for transient simulations of TII (140-127 kyr BP) under the auspices of PMIP4, as well as a selection of records, providing appropriate benchmarks for subsequent model-data comparisons (Menviel et al. 2019).

Our understanding of glacial-interglacial cycles has been built on a large body of evidence from Middle and Late Pleistocene environments, dominated by $100-k y r$ icevolume variations. However, any theory of ice ages remains incomplete if it does not include an adequate description and understanding of the mode and tempo of climate variability during the Early Pleistocene (the so-called "41-kyr world") and the transition into the "100-kyr world" (Mid-Pleistocene Transition, MPT). With this in mind, the third QUIGS workshop (28-30 September 2017; pastglobalchanges.org/calendar/2017/127pages/1655) explored the characteristics of interglacials of the 41-kyr world and considered causes of the MPT (Ford and 
Chalk 2020). Despite the emergence of boron-based $\mathrm{CO}_{2}$ data from marine cores, progress in modeling 41-kyr cycles in sea level and the causes of the MPT requires $\mathrm{CO}_{2}$ reconstructions with reduced uncertainties. The planned drilling of an "Oldest Ice" core back to 1.5 million years ago (Myr BP) will eventually provide increased confidence on the evolution of the climate-carbon cycle interactions for this period.

One of the outstanding questions identified by PIGS in their final review paper was: "Given the astronomical forcing and the feedbacks that are present, is the occurrence and character of interglacials predictable? In other words, ... is it inevitable that we would find ourselves in today's interglacial climate following the same sequence of glacial and interglacials that has occurred?" (Past Interglacials Working Group of PAGES 2016, p. 206). An initial answer to this was provided by Tzedakis et al. (2017), who proposed that an interglacial onset occurs when a peak in insolation exceeds a threshold that decreases with time elapsed since the previous deglaciation, as ice sheets become more unstable. This correctly predicted the deglaciation history during the Quaternary and identified a gradual rise in the deglaciation threshold from 1.5 Myr BP that led to an increase in the frequency of skipped insolation peaks after 1 Myr BP (Fig. 2). The emergence of longer glacials then allowed the accumulation of larger and increasingly unstable ice sheets. The analysis also showed that the succession of interglacials is not chaotic; the sequence that has occurred is one among a very small set of possibilities, suggesting a degree of probabilistic determinism.

Between Phases 1 and 2, QUIGS and another PAGES working group, PALeo constraints on SEA-level rise (PALSEA), held a joint workshop from 24-27 September 2018 (pastglobalchanges.org/calendar/2018/127pages/1759). The goal of the workshop was to identify the state of our understanding on the interplay between climate, polar ice sheets, and sea level during past interglacial periods. A position paper identified eight research areas as critical for an improved understanding of climate and ice-sheet responses to astronomical and greenhouse gas forcing, and by extension, responses to conditions similar to or warmer than the preindustrial climate (Capron et al. 2019).

2019 marked the start of the second phase of QUIGS in which improved datasets and new model experiments are being used to address research questions and knowledge gaps identified during Phase 1. A workshop on "Warm extremes - MIS $5 \mathrm{e}$ and its relevance to the future" (1-4 July 2019; pastglobalchanges.org/calendar/2019/127pages/1910) played a key role in focusing community efforts (model and data) to publish relevant science for the forthcoming 6th Assessment Report of the IPCC. In particular, papers comparing the ensemble of new CMIP6-PMIP4 lig127k simulations and proxy reconstructions of surface temperature and sea ice were developed (Kageyama et al. 2021; Otto-Bliesner et al. 2021). The latter

(a) $40^{\circ}-90^{\circ} \mathrm{N} \mathrm{JJA}$

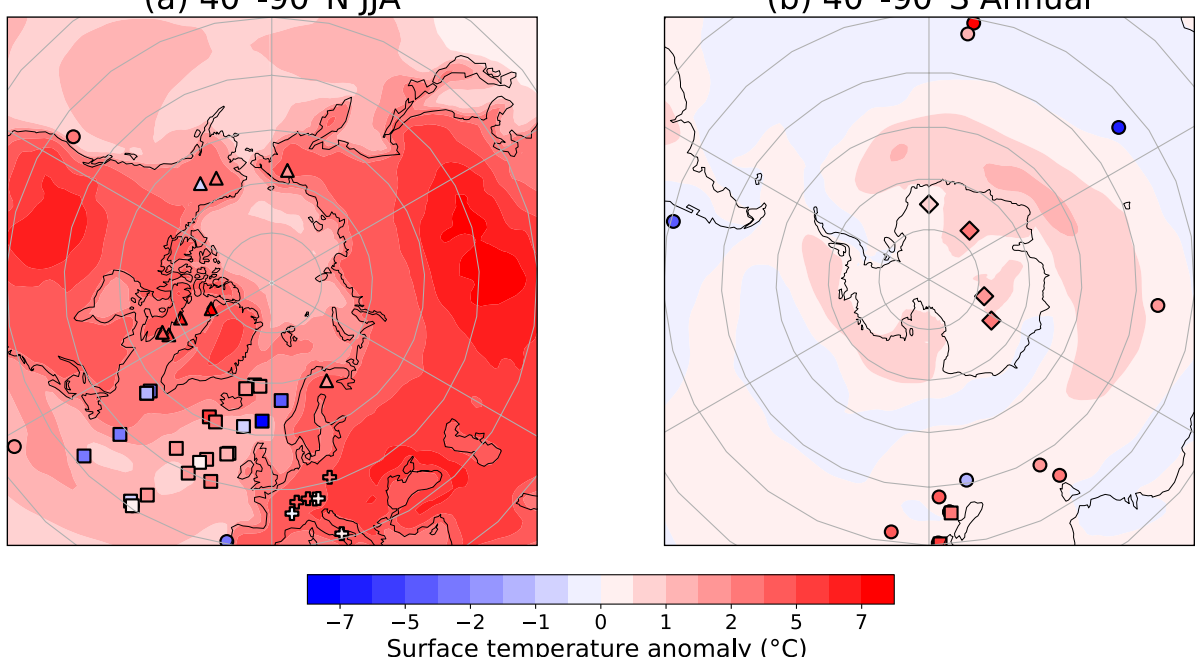

Surface temperature anomaly $\left({ }^{\circ} \mathrm{C}\right)$

Figure 3: Comparison of results of the CMIP6-PMIP4 lig127k simulations and proxy records. High-latitude surface temperature anomaly comparing $127 \mathrm{kyr}$ BP to the preindustrial period from models (ensemble average in colors) and proxies (circles for the compilation by Hoffman et al. (2017); squares and diamonds for marine sites and ice cores, respectively, from the compilation by Capron et al. $(2014,2017)$; pluses for the compilation of Brewer et al. (2008); and triangles for the Arctic compilation, https://doi.org/10.5194/cp-17-63-2021-supplement): (A) $40^{\circ}-90^{\circ} \mathrm{N}$ June-July-August, (B) $40^{\circ}-90^{\circ} \mathrm{S}$ annual. The preindustrial reference is $1850 \mathrm{CE}$ for model anomalies and for the data is 1870-1899. Modified from Otto-Bliesner et al. (2021), courtesy of A. Zhao.

paper showed that the model ensemble was able to simulate the reconstructed $127-\mathrm{kyr}$ BP JJA temperature anomalies over Canada, Scandinavia, parts of midlatitude Europe, and much of the North Atlantic (Fig. 3). The exceptions are in the northwestern North Atlantic and Nordic seas, where the marine reconstruction suggests significant cooling (Capron et al. 2017). Potential reasons for mismatches include dating uncertainties, a lingering memory of the $\mathrm{H} 11$ event in marine records (Marino et al. 2015), and/or the design of the CMIP6-PMIP4 lig127k protocol without meltwater from potential remnant ice sheets over Canada and Scandinavia.

In the midst of the COVID-19 pandemic, a virtual meeting on glacial termination processes and feedbacks was held on 10 and 12 November 2020 (pastglobalchanges. org/calendar/2016/127-pages/1592). Seventyfive percent of the talks were given by early career researchers (ECRs), who presented advances in understanding of deglacial changes in climate, ice sheets, the carbon cycle, and vegetation. Further meetings on terminations and the MPT are planned. A final workshop on one of the most challenging issues, the causes of interglacial intensity, will provide a fitting close to the PAGES interglacial effort.

\section{Coda}

Looking back from today's perspective of accelerating global warming, initiating a working group with a specific focus on interglacials appears the obvious thing to have done. But in January 2007, with much attention centered on glacial climate variability, this was not necessarily obvious. From a small group of friends, the PAGES interglacial community grew to involve 69 (PIGS) and 95 (QUIGS) scientists, while ECR participation increased from $10 \%$ to over $40 \%$. We are grateful to PAGES, and especially Thorsten Kiefer and Marie-France Loutre, for their encouragement and continued support in this endeavor. It has been a fabulous ride.

\section{AFFILIATIONS}

${ }^{1}$ Environmental Change Research Centre, Department of Geography, University College London, UK ${ }^{2}$ Climate Change Research, Centre, PANGEA, University of New South Wales, Sydney, Australia ${ }^{3}$ Institut des Géosciences de l'Environnement, CNRS, Grenoble, France

${ }^{4}$ Climate and Global Dynamics Laboratory, National Center for Atmospheric Research, Boulder, CO, USA ${ }^{5}$ Lamont-Doherty Earth Observatory, Columbia University, Palisades, NY, USA

${ }^{6}$ Department of Earth Sciences, University of Cambridge, UK

\section{CONTACT}

Chronis Tzedakis: p.c.tzedakis@ucl.ac.uk

\section{REFERENCES}

Berger A (1978) J Atmos Sci 35: 2362-2367

Brewer S et al. (2008) Quat Sci Rev 27: 2303-2315

Capron E et al. (2014) Quat Sci Rev 103: 116-133

Capron E et al. (2017) Quat Sci Rev 168: 137-150

Capron E et al. (2019) Quat Sci Rev 219: 308-311

Cheng $\mathrm{H}$ et al. (2009) Science 326: 248-252

Ford HL, Chalk TB (2020) Oceanography 33:101-103

Hoffman JS et al. (2017) Science 355: 276-279

Jouzel J et al. (2007) Science 317: 793-796

Kageyama M et al. (2021) Clim Past 17: 37-62

Lisiecki LE, Raymo ME (2005) Paleoceanography 20: PA1003

Lüthi D et al. (2008) Nature 453: 379-382

Marino G et al. (2015) Nature 522: 197-201

Menviel L et al. (2019) Geosci Model Dev 12: 3649-3685

Mokeddem Z et al. (2014) Proc Natl Acad Sci USA 111: $11,263-11,268$

Otto-Bliesner BL et al. (2017) Geosci Model Dev 10: 3979-4003

Otto-Bliesner BL et al. (2021) Clim Past 17: 63-94

Past Interglacials Working Group of PAGES (2016) Rev Geophys 54: 162-219

Tzedakis PC et al. (2009) Nat Geosci 2: 751-755 Tzedakis PC et al. (2012) Clim Past 8: 1473-1485 Tzedakis PC et al. (2017) Nature 542: 427-432 